\title{
A DiSCRETE ANALOGUE OF THE PALEY-WIENER THEOREM FOR BOUNDED ANALYTIC FUNCTIONS IN A HALF PLANE
}

\author{
DORON ZEILBERGER
}

(Received 8 September 1976)

Communicated by E. Strzelecki

In this note we prove a discrete analogue to the following Paley-Weiner theorem: Let $f$ be the restriction to the line of a bounded analytic function in the upper half plane; then the spectrum of $f$ is contained in $[0, \infty)$. The discrete analogue of complex analysis is the theory of discrete analytic functions invented by Lelong-Ferrand (1944) and developed by Duffin (1956) and others. A function $f$ on a subset $M$ of the two-dimensional lat tice $Z^{2}$ is said to be discrete analytic there if, for $(m, n) \in M$,

$$
[f(m+1, n+1)-f(m, n)] / 1+i=[f(m, n+1)-f(m+1, n)] / i-1
$$

which is equivalent to the requirement

$$
f(m, n)+i f(m+1, n)-f(m+1, n+1)-i f(m, n+1)=0 .
$$

Given a bounded sequence $\left\{c_{n}\right\}_{-\infty}^{\infty}$, its spectrum is the support of the distribution $\Sigma_{-\infty}^{\infty} c_{n} e^{- \text {int }}$ on the circle $T$. It is well known that it coincides with the Fourier-Carleman spectrum which is the complement of the set through which

$$
\sum_{0}^{\infty} c_{n} z^{-n}(|z|>1) \quad \text { and } \quad-\sum_{-\infty}^{-1} c_{n} z^{-n} \quad(|z|<1)
$$

can be analytically continued to each other.

Now, we are in a position to prove the following theorem.

THEOREM: Let $f(m, n)$ be discrete analytic and bounded in the upper half lattice, $n \geqq 0$. The spectrum of $\{f(m, 0)\}_{-\infty}^{\infty}$ is contained in $[0, \pi)$.

Proof: The theorem is obviously true for $f$ constant, for then $\Sigma_{-\infty}^{\infty} C e^{-\mathrm{int}}=C \delta, \delta$ being the Dirac delta measure, which is supported at $\{0\}$. 
So without loss of generality we may assume that $f(0,0)=0$. The theorem will be proven if we show that

$$
\sum_{0}^{\infty} f(m, 0) z^{-m} \quad(|z|>1) \quad \text { and } \quad-\sum_{-\infty}^{-1} f(m, 0) z^{-m} \quad(|z|<1)
$$

can be analytically continued to each other through the lower unit semi-circle; or, equivalently that $\varphi_{+}(z)=\sum_{0}^{x} f(m, 0) z^{m} \quad(|z|<1)$ and $-\varphi_{-}(z)=$ $-\Sigma_{-\infty}^{-1} f(m, 0) z^{m}(|z|>1)$ are analytic continuations of each other through the upper unit semi-circle.

Let

$$
F(z, w)=\sum_{n=0}^{\infty} \sum_{m=0}^{\infty} f(m, n) z^{m} w^{n}, \quad \psi(w)=\sum_{n=0}^{\infty} f(0, n) w^{n} .
$$

Since $f$ is bounded, $F, \varphi_{+}, \psi$, are all analytic in the polydisc $\{|z|<1\} \times$ $\{|w|<1\}$. By (1),

$$
(1+i z-z w-i w) F(z, w)=(1+i z) \varphi_{+}(z)+(1-i w) \psi(w)
$$

and it follows that the r.h.s. vanishes for $|z|<1,|w|<1, w=(1+i z) /(z+i)$. Therefore, for $|z|<1,\left|\frac{z-i}{z+i}\right|<1$,

$$
\varphi_{+}(z)=\frac{2 i z}{z^{2}+1} \psi\left(\frac{1+i z}{z+i}\right)
$$

Since $\psi(0)=0$, the r.h.s. of (3) has no pole at $z=i$ and is an analytic function in the upper half plane $\left\{\left|\frac{z-i}{z+i}\right|<1\right\}$. It follows that $\varphi_{+}(z)$ can be analytically continued through the upper semi-circle to the whole upper half plane. A similar reasoning for the left upper quarter lattice shows that

$$
\varphi_{-}(z)=\frac{-2 i z}{z^{2}+1} \psi\left(\frac{1+i z}{z+i}\right) \quad\left(|z|>1,\left|\frac{z-i}{z+i}\right|<1\right) .
$$

Thus $\varphi_{-}(z)$ can be analytically continued through the upper unit semi-circle to the whole upper half plane and we have that $\varphi_{+}(z)$ and $-\varphi_{-}(z)$ are analytic continuations of each other through the upper unit semi-circle and the theorem follows.

\section{Acknowledgement}

The author wishes to thank Perez Amir for helpful comments and discussions. 


\section{References}

J. Ferrand (1944). 'Fonctions pre'harmonique et fonctions pre'holomorphees'. Bull. Sci. Math.

68, 152-180.

R. J. Duffin (1956), 'Basic properties of discrete analytic functions'. Duke Math. J. 23, 335-363.

Department of Mathematics,

The Weizmann Institute of Science,

Rehovot, Israel.

Current postal address: School of Mathematics, Institute for Advanced Study, Princeton, N.J. 08540. 